\title{
Smart Grid Protection through Self-Healing
}

\author{
Chathurika Chandraratne, R.T. Naayagi, Thillainathan Logenthiran \\ School of Electrical and Electronic Engineering \\ Newcastle University (Singapore) \\ Singapore 567739 \\ E-mails: C.S.K.Chandraratne2@newcastle.ac.uk; naayagi.ramasamy@ncl.ac.uk; t.logenthiran@ncl.ac.uk
}

\begin{abstract}
Modern power system is more flexible and complex than the conventional power system. From a reliability point of view it is important to identify and isolate the fault as fast as possible from the neighbouring zones. This paper proposes protection solutions for smart grid. In particular, the main focus of this paper is on three different protections namely overcurrent protection, transformer protection, and directional overcurrent protection. The proposed protection solutions are validated through IEEE- 9 bus and 14 bus electrical power systems. ETAP software has been used to verify the system operation.
\end{abstract}

Keywords-Traditional power grid; smart grid; Overcurrent relay; Directional protection; Differential transformer relay protection; Self-healing

\section{INTRODUCTION}

In recent years, there has been a tremendous increase in the deployment of renewable energy resources in electrical power system. This is because of increased environmental concerns and global climate change. Europe and many other countries have started to construct smart grids. They consider this as an important part of their national power strategy [1] [2]. Today, smart grid has named as the next generation of the power grid which uses two-way communication of electricity and digital technology to supply electricity. Smart grid improves the reliability and reduces the peak demand. It also helps to increase the energy efficiency along with the environmental benefits gained by such efficiency. However, it poses a number of challenges to electrical engineers in all aspects especially in developing appropriate protection solutions because of the bidirectional power flow capability.

Conventional power system uses various protective relays to isolate a device or a system during a fault. However, in some cases if the relay is over sensitive it may cause unexpected trips which could cause cascading failures [3] - [5]. In this paper, self-healing is applied to improve the reliability to avoid false trips thereby preventing the disturbances from spreading across the entire power grid. In a smart grid, the computer and communication systems will send corrective measures in less than half a second in the case of a fault thereby triggering the circuit breaker to isolate the faulty line, and this prevents other lines from being damaged [6] - [10].

\section{TYPES OF PROTECTION}

\section{A. Background}

The smart protection system is the subsystem in a smart grid that provides advanced grid reliability analysis, failure protection, and security and privacy protection services [11]. By taking advantage of the smart infrastructure, the smart grid must not only realize a smarter management system, but also provide a smarter protection system which can more effectively and efficiently support failure protection mechanisms, address cyber security issues, and preserve privacy. Protective relays are designed in such a way that it senses and isolates a fault quickly without disrupting the unaffected areas. It is important to the industry because it helps to prevent large production losses due to equipment damage resulting from a fault or overload. This also helps to take care of people's safety, and any timely replacement of equipment to avoid any losses [12]. Different relays have different protections, for example an overcurrent relay senses a current when it is higher than the actual value, and then it will trip. It is important to follow the criteria while selecting a protection solution. Firstly, it should be cost effective and should be able to give maximum protection to the system. Secondly, it should be reliable and should be able to facilitate a high-speed operation. Most importantly, when a fault occurs the protection should act fast to isolate the fault while the neighboring areas will run smoothly. Reliability, selectivity, speed and sensitivity are key attributes to have a good performance of a relay in a power system. Another important aspect is the selection of the current transformer ratio. The nominal and short circuit currents of the power network play a huge part in the current transformer ratio choice. This ratio is determined by the magnitude of the nominal and short circuit currents with no saturation. It is calculated as

so that

$$
\mathrm{I}_{\mathrm{SC}}(5 / \mathrm{X}) \leq 100(\mathrm{~A})
$$

$$
X \geq(5 / 100)\left(I_{S C}\right)
$$

$\mathrm{I}_{\mathrm{SC}}$ represents the short circuit current and $\mathrm{X}$ represents the current constant. Another way to determine the current transformer ratio is by looking at the rated current of the cable and the transformer which are used in the system. 


\section{B. Zone of protection}

Protection zones are considered for primary and/or backup protection. The primary protective relays are the first line of defense against system faults and will be the first to isolate the fault [13]. If it fails to isolate, the backup protection will be activated. It is done by re-tripping the primary circuit breaker. Another way is to trip the circuit breakers which are closer to the fault zone [14]. There are two types of backup protection, and they can be either local or remote. Local backup protection is located at the same zone in which the fault occurs. When a fault occurs, the circuit breaker will trip either the primary or the nearby zones. The remote backup protection is located at the nearby zones and will only trip in that zone. The basic requirement to protect the whole system is by overlapping the neighboring zones. By doing so, no part of the system is unprotected. However, when a fault happens, both the overlapped zones will be isolated. Therefore, overlapping of zones should be minimized as much as possible to reduce the power distruption in the unaffected zones.

\section{Overcurrent Relay Protection}

Overcurrent relay is mostly used to protect the system components in a radial distribution. It is also one of the easiest types of protection. Figure 1 represents the working of the overcurrent relay.

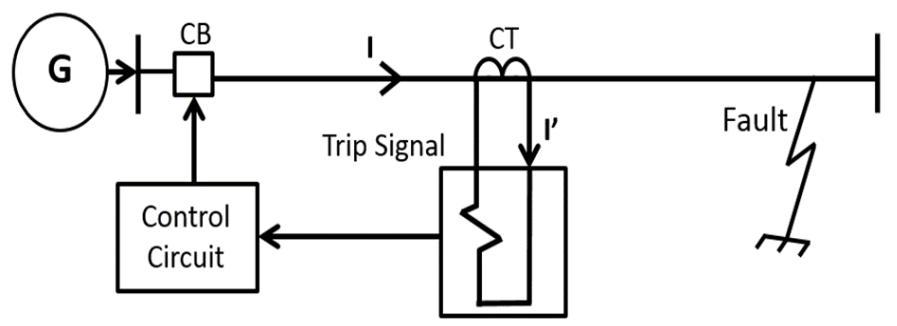

Figure 1: Representation of overcurrent relay

The current I' from the secondary side of the current transformer is from the relay signal, while the pick-up or the threshold current $\mathrm{I}_{\text {pickup }}$ is from the relay setting.

Operating Principle:

Under normal operation: I' $<\mathrm{I}_{\text {pickup }}$

Short Circuit fault: I'> $\mathrm{I}_{\text {pickup }}$

Under normal operation, there is no trip signal therefore the circuit breaker (CB) is closed. When the secondary current of the transformer is greater than the pick-up current, there is a trip signal and therefore $\mathrm{CB}$ will open and isolate the line. To protect a system, the pick-up and time-dial setting of relays must be properly selected and accurately set based on the required operating times and the fault currents. The procedure of selecting and setting protective devices is called protection coordination. There are two types of overcurrent relays. They are instantaneous overcurrent relay and time-overcurrent relay. Instantaneous overcurrent relay is designed in such a way that there is no time delay in the operation when it goes beyond the relay setting. The operational time can change extremely. The timing can go as low as 0.016 seconds or to 0.1 seconds high [4] while time-overcurrent relay has an operating characteristic such that its operating time varies inversely. Under timeovercurrent relay, there are three commonly used characteristics. As the operating time decreases, the current increases. They are inverse, very inverse, and extremely inverse.

\section{Directional Relay Protection}

The directional overcurrent relay is represented by Relay 67 as per IEEE standard. The directional protection has a similar principle as that of overcurrent protection except that it has a function of choosing either the forward or the reverse direction. This type of protection is mainly used in parallel lines. It can be also used for both ground and phase faults. The values that are necessary for this type of protection are current, voltage, and the angle between current and voltage. An Intelligent Electronic Device, (IED) is needed to compare the system's line current with fault current to find the direction of the fault. This is called polarizing quality [3]. When a ground fault occurs, the residual voltage is equal to three times the zero-sequence voltage drops on the source impedance. The residual voltage is zero when the phase voltages are balanced. This may cause the displacement from the residual current by the characteristic angle of the source impedance. The values must be calculated correctly to have the correct directional protection.

There are ways when a false tripping may happen. One example is improper coordination and protection modification when a distributed generator is connected to the medium voltage feeder. Though it will trip correctly based on the shortcircuit fault but the neighboring $\mathrm{CB}$ with the distributed generator (DG) will trip as well. This type of incorrect tripping is common when the synchronous generation is used as a DG source. It can be fixed by using directional protection [6]. The directional relay recognizes the direction in which the fault occurs relative to the location of the relay. It is set such that it is activated when a fault occurrs in one direction only. It is not activated for faults occurring in the other direction.

\section{E. Transformer Differential Relay Protection}

The differential transformer relay protection is represented by 87T. Differential relay protection follows the concept of Kirchhoff's current law. It means that the sum of the currents entering the transformer is equal to the sum of the currents leaving it. Therefore, there will be no differential current. The relay works when the differential current is greater than zero [4]. Differential protection not only protects the internal fault of the transformer but also the internal faults of large motors, generators, lines or cables and bus. However, it does not protect faults due to overload or external faults. If necessary to protect the system from external faults, an overcurrent relay is needed.

\section{F. Self-healing smart grid}

To achieve self-healing in a power grid, the system should have sensors, automated controls, and advanced software that uses real-time distribution of data to detect and the isolate faults and to reconfigure the distribution network to minimize the power 


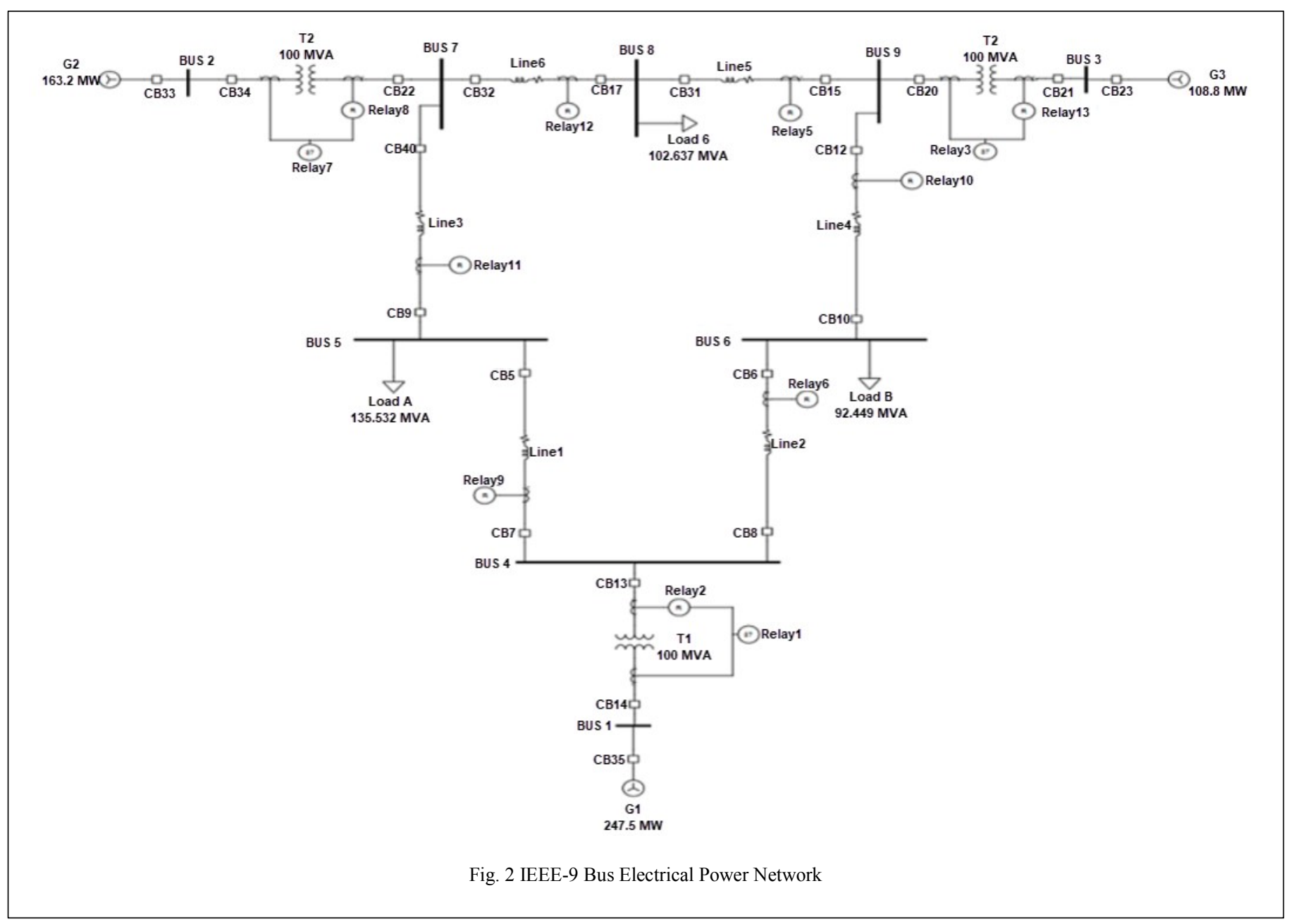

outage and customer impact [15]. One of the key objectives of a self-healing grid is to improve the system reliability. This can be accomplished by reconfiguring the $\mathrm{CBs}$ and relays and reclosers installed on the distribution feeder to quickly isolate the faulty section of the feeder and re-establish the service to as many customers as possible from alternate sources or feeders. Self-healing solutions typically need to reconfigure the system within one to five minutes. To accomplish this high bandwidth communications may be required.

Self-healing is the property that enables a system to perceive if a system is not operating correctly and, without human intervention, make necessary adjustments to restore the system by itself to normality. Self-healing usually refers to reconfiguration, load shedding, and controlling the generators' output powers. Self-healing actions are a multi-objective, nonlinear optimization problem with a number of constraints. Advanced algorithms need to be developed using artificial intelligence techniques and multi-agent systems to solve these problems. Restoration problems are much sophisticated in smart grids due to the challenges such as bidirectional power flow, mesh connected topologies, and limited capacities of distributed energy resources (DERs).
The following explains the deployment of self-healing with the utility and consumer side. Some of these functions are also present in a normal grid.

Distribution Automation: This automatically reroutes power based on the load requirements, the generation level and fault conditions. Due to the communication feature of smart grid this facilitates to make intelligent decisions.

Reclosers: In a normal grid, when there is a fault the $\mathrm{CB}$ will open and after sometime the $\mathrm{CB}$ will reconnect to check whether the fault still persists in the system. But in a smart grid, the recloser will report the failure over the communication network and wait for further instructions.

Fault circuit indicators: These monitor the power lines and feeders by constantly sensing the voltage, current and fault conditions. In the case of a fault, it will communicate this information immediately for corrective actions to take place.

\section{SIMULATION STUDIES AND RESULTS}

Simulation was performed using the ETAP software. The results are based on IEEE-9 bus and IEEE-14 bus electrical power networks. Firstly, load flow analysis is performed followed by the short circuit analysis in which the network is subjected to three phase fault currents. Finally, the protection 
solutions are introduced. The protection that have been applied in these systems are overcurrent relay protection, differential transformer relay protection, and directional overcurrent relay protection.

\section{A. IEEE-9 BUS SYSTEM}

The IEEE-9 bus electrical power network is shown in Figure 2. This network consist of nine buses, three generators, three twowinding power transformers, six lines and three loads. It has a three way electricity flow as it has three generators to power up the system. Figure 3 and 4 depict the simulation results for over current protection when a fault occurs between Bus 4 and Bus 6 and an internal protection activation when there is an internal fault in the transformer 1 .

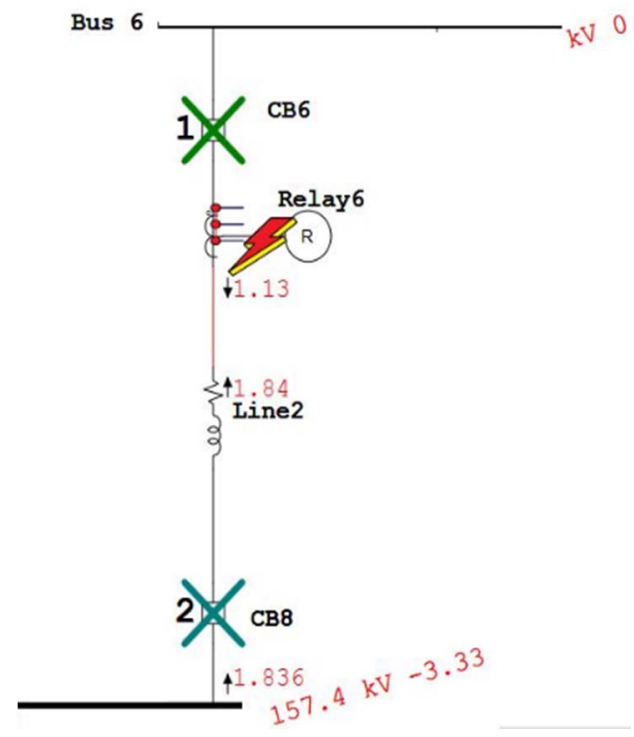

Fig.3 Simulation results of Overcurrent Protection

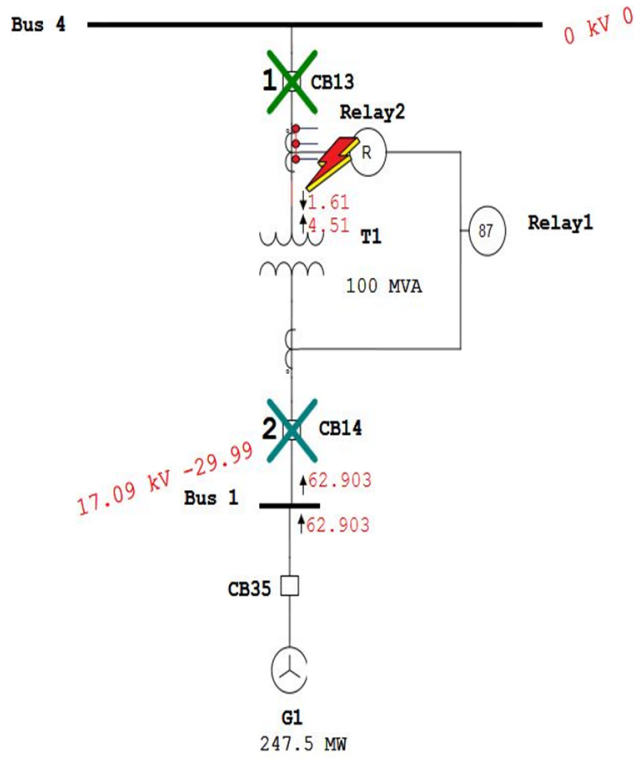

Fig.4 Simulation results for Internal Protection
TABLE 1. TRIPPING SEQUENCES BETWEEN TWO BUSES

\begin{tabular}{|l|l|l|}
\hline Bus Number & Relay (®) & Backup \\
\hline Bus4 \& Bus6 & CB6 \& CB8 & CB10 \\
\hline Bus4 \& Bus5 & CB5 \& CB7 & CB9 \\
\hline Bus5 \& Bus7 & CB9 \& CB40 & CB22 \\
\hline Bus6 \& Bus9 & CB12 \& CB10 & CB15 \\
\hline Bus7 \& Bus8 & CB17 \& CB32 & CB22 \\
\hline Bus8 \& Bus9 & CB15 \& CB31 & CB17 \\
\hline
\end{tabular}

TABLE 2. TRIPPING SEQUENCES FOR TRANSFORMERS

\begin{tabular}{|c|l|l|l|}
\hline Transformer & Relay (87) & Relay ${ }^{\circledR}$ & Backup \\
\hline T1 & CB13 & CB13 & CB35 \\
(Bus1 \& B us4) & CB14 & CB14 & \\
\hline T2 & CB22 & CB22 & CB33 \\
(Bus2 \& Bus7) & CB34 & CB34 & \\
\hline T3 & CB20 & CB20 & CB22 \\
(Bus9 \& Bus3) & CB21 & CB21 & \\
\hline
\end{tabular}

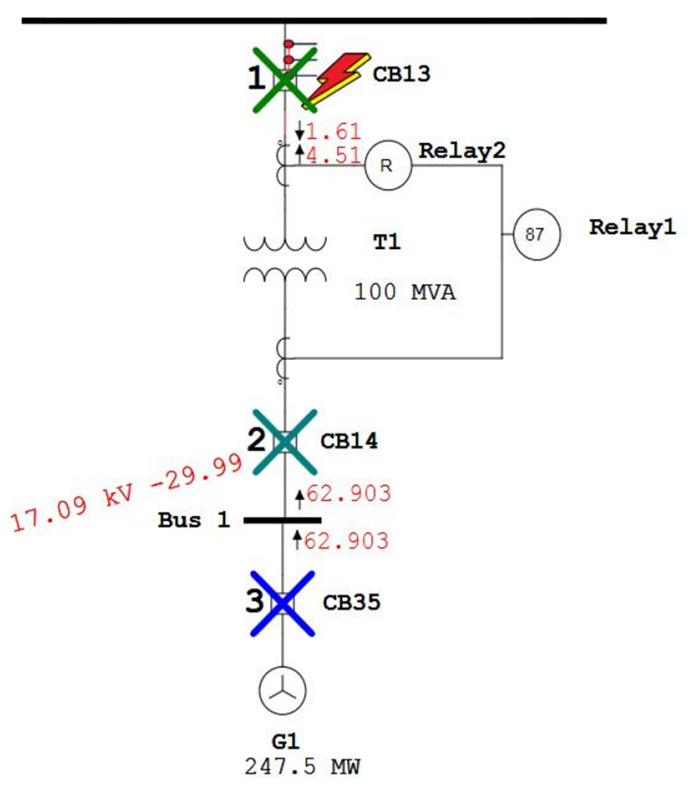

Fig. 5 Simulation results for External Protection

In order, to protect external faults an overcurrent current is combined with Relay1. Therefore, CB13, CB14, and CB35 will trip as shown in Figure 5.

Tables 1 and 2 give the results of tripping sequences when a fault occurs in the system. Self-healing concept is applied to minimize the power distruption and improve the reliability. Employing self-healing in a smart grid helps to locate the fault faster and isolate the affected area so that electricity can be restored to the unaffected areas.

\section{B. IEEE-14 BUS SYSTEM}

The following circuit is a small part of the American Electrical Power Network. This network consist of 14 buses, five generators, three two-winding power transformers, six lines and 11 loads. It has five ways of electricity flow as it has five generators to power the system. For illustration purpose, the protection is incorporated in the three transformers. Bus 1 and 


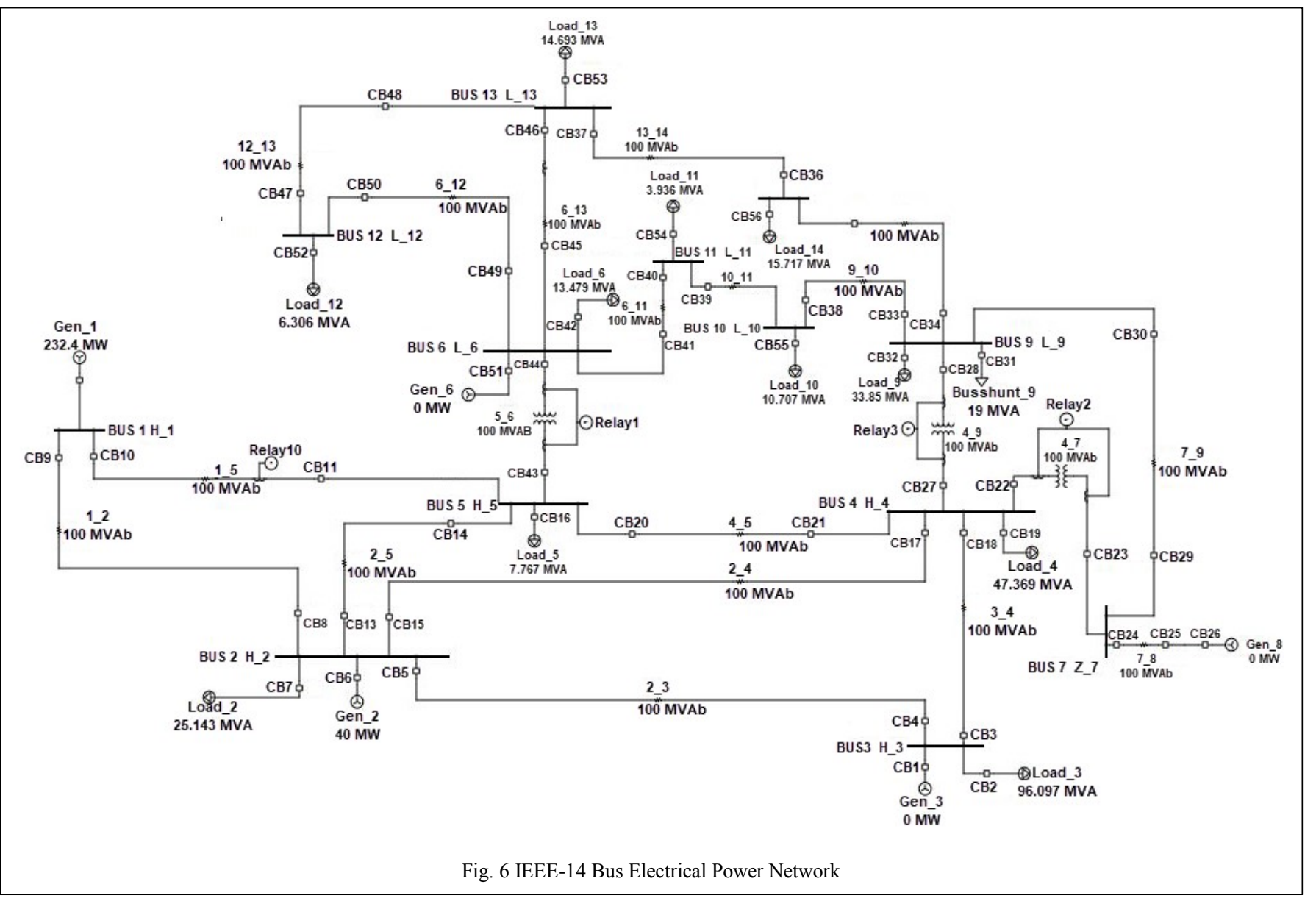

TABLE 3. TRIPPING SEQUENCES FOR TRANSFORMERS

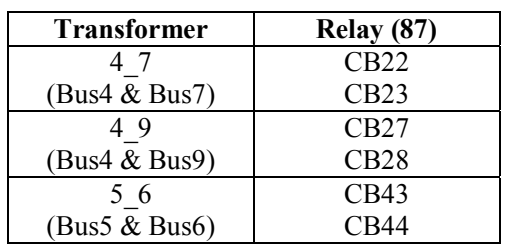

Bus 5 are protected by employing the self-healing concept. The best kind of protection to be used in this network is directional overcurrent protection. It is because one bus has more than one connection from other buses. Also, directional protection can control which direction the CB should trip when a fault occurs. The results obtained from the ETAP simulations are shown in Table 3. If a fault occurs between Bus 1 and Bus 5, CB10 and CB11 would trip.

\section{CONCLUSION}

This paper presented the protection solutions for a smart grid. In particular, self-healing has been employed for IEEE 9 bus and IEEE 14 bus electrical power networks. The electrical power networks were modelled using the ETAP simulation environment. Three different protections such as overcurrent protection, transformer protection and directional overcurrent protection have been applied for various types of faults. The proposed protection solutions are validated through IEEE- 9 bus and 14 bus electrical power systems. Simulation results obtained from the ETAP software verifies the effectiveness of self-healing in a modern power system operation.

\section{REFERENCES}

[1] Claudio Sergio Mardegan, Rasheek Rifaat, "Considerations in Applying IEEE Recommended Practice for Protection Coordination in Industrial and Commercial Power Systems-Part I", IEEE Transactions on Industry Applications , vol.52, no.5, pp. 3705-3713, 2016.

[2] Ayaz Ahmad and Naveed Ul Hassan, "Smart Grid as a Solution for Renewable and Efficient Energy", April 2016.

[3] Distribution System Feeder Overcurrent Protection, GE Power management report-GET-6450, 1-21, Last accessed May 2017.

[4] Michael Stanbury, Zarko Djekic, "The Impact of Current-Transformer Saturation on Transformer Differential Protection", IEEE Transactions on Power Delivery, vol.30, no.3, pp. 1278-1287, 2015.

[5] Muhammad Ramadan, Edwin Chiang Jia Hao, Thillainathan Logenthiran, R.T. Naayagi, W.L. Woo, "Islanding Detection of Distributed Generator in Presence of Fault Events," in proceedings of the IEEE Region 10 Conference (TENCON), November 5-8, 2017, Penang, Malaysia.

[6] D. Novosel, M. Begovic, and V. Madan. Shedding light on blackouts. IEEE Power and Energy Magazine, Jan. 2004.

[7] J. Liu, Y. Xiao, S. Li, W. Liang, and C. L. Chen. Cyber security and 
privacy issues in smart grids. Communications Surveys \& Tutorials, IEEE, 14(4):981-997, 2012.

[8] Muhammad Ramadan, Surian Raj, T. Logenthiran, R.T. Naayagi, W.L. Woo, "Self-healing Network Instigated By Distributed Energy Resources," in proceedings of the $9^{\text {th }}$ IEEE PES Asia-Pacific Power and Energy Engineering Conference, November 8-10, 2017, Bangalore, India.

[9] B.M.S. Muhammad Ramadan, T. Logenthiran, R.T. Naayagi, C. Su, "Accelerated Lambda Iteration Method for Solving Economic Dispatch with Transmission Line Losses Management," in proceedings of IEEE PES Innovative Smart Grid Technologies (ISGT Asia) Conference, pp. 138-143, 2016, Melbourne, Australia.

[10] T. Logenthiran, R.T. Naayagi, W.L. Woo, Van-Tung Phan, K. Abidi, "Intelligent Control System for Microgrids using Multi-Agent System," IEEE Journal of Emerging and Selected Topics in Power Electronics, vol. 3, no. 4, pp. 1036-1045, December 2015.
[11] H. Farhangi., The path of the smart grid, IEEE Power and Energy Magazine, Vol.8 , Issue.1, 2010.

[12] J. Zhang and Y. Dong. Preventing false trips of zone 3 protection relays in smart grid. Tsinghua Science and Technology, 20(2):142-154, 2015.

[13] S. Garlapati, H. Lin, S. Sambamoorthy, S. K. Shukla, and J. S. Thorp. Agent based supervision of zone 3 relays to prevent hidden failure based tripping, 2010 First IEEE International Conference on Smart grid communications, pages 256-261. IEEE, 2010.

[14] Jiapeng Zhang, Yingfei Dong, "Power-Aware Communication Management for Reliable Remote Relay Protection in Smart Grid", IEEE Power Systems Conference, pp.1-6, 2016.

[15] J. Gao, Y. Xiao, J. Liu, W. Liang, and C. L. P. Chen, "A survey of communication/networking in smart grids", Future Generation Computer Systems, 28(2):391-404, 2012. 